International Journal of Pure and Applied Mathematics

Volume 111 No. 1 2016, 105-119

ISSN: 1311-8080 (printed version); ISSN: 1314-3395 (on-line version)

url: http://www.ijpam.eu

doi: 10.12732/ijpam.v111i1.10

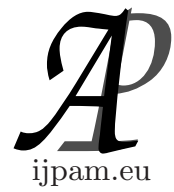

\title{
RESPONSIVE USER INTERFACE FOR PEOPLE WITH ASD
}

\author{
Nikolay Pavlov ${ }^{1}$, Kliment Mirchev ${ }^{2}$, Teodora Gardjeva ${ }^{3}$, \\ Dilyana Krushkova ${ }^{4}$, Asen Rahnev ${ }^{5} \S$ \\ ${ }_{1,3,4}$ Plovdiv University \\ 236, Bulgaria, Blvd., Plovdiv 4000, BULGARIA \\ ${ }^{2} 9 \mathrm{~N}$ Kuklensko shose, Blvd., Plovdiv 4000, Bulgaria \\ ${ }^{4}$ 11-ti Avgust, Str., Plovdiv 4000, BULGARIA
}

\begin{abstract}
This paper describes the design and implementation of the user interface of Open Book, a reading assistive tool for people with autism spectrum disorders (ASD). The findings in existing research played a vital role in UI implementation. Key screens of the user interface with the main features and functionality of the system are presented. The conducted evaluation by users with ASD and their caregivers clearly proves the usability and benefits of the innovative software product.
\end{abstract}

Key Words: responsive user interface, accessible user interface, users with autism spectrum disorders, project first, open book

\section{Introduction}

This paper describes the design and implementation of the user interface of Open Book. Open Book is a distributed software system employing various natural language processing components to convert documents for people with (Autistic Spectrum Disorders) ASD in a format easier to read and understand. Open Book is developed as a part of project FIRST (A Flexible Interactive

Received: $\quad$ August 11, 2016

Revised: $\quad$ October 23, 2016

Published: December 6, 2016

$\S$ Correspondence author
(C) 2016 Academic Publications, Ltd. url: www.acadpubl.eu 
Reading Support Tool) partially funded by the European Commission under the Seventh Framework Programme (FP7-2007-2013) for Research and Technological Development under grant agreement \# 287607.

The FIRST project starts from the need to improve the life of people with ASD by enabling them to read any written documents and thus improving their social inclusion and interaction. People with ASD are approximately 3.3 million across Europe according to Autism Speaks research (see [2]). People with ASD may experience difficulties comprehending complex instructions while reading, getting misled by figurative language or the use of rare words or, simply, get distracted by secondary points touched upon in a document (see [1]). Using innovative language technology to simplify documents, the created software product Open Book helps ASD users convert a standard document into a personalized version which is easier for them to read and understand.

\section{Primary Features of Open Book}

A quick review of the Open Book's first prototype basic features will help comprehend better the user interface of the tool, presented later on in this paper in section Design Implementation. This is the first version of the tool and therefore the feature set is subject to further development and change.

The system provides the following functions to users with ASD:

- Simplification - the text entered by user is automatically converted to a simpler and convenient format. If user still finds some word or expressions difficult, he can ask the system for explanation, additional information or some images;

- Personalization - user can adjust style, font and format of the simplified text according to his personal preferences and requirements. The system provides a set of specially designed by the clinical partners color themes which make the document reads better. However, user can also create his own color themes which best fulfill his special needs;

- Improvement of readability - for user's convenience the system provides features like highlighter and magnifier notes which help user to go less effortlessly through the whole text or leave his thoughts and comments by using notes;

- Better organization of documents - user can arrange his documents as he prefers - divide them into folders by using labels, mark them as ready or favorite, search and find easily the desired document and others; 
- Communication with the caregiver - if user still has problems with the document content after simplification, he can send notification to his carer sharing his problems and requirements for a particular document;

- Preferences and selection of tools - user can select the default theme, font, create labels for the library organization and choose the most useful tools for his personal toolbar;

\section{Approach to Design}

Open Book is not just being developed for people who have ASD, it is being developed with people who have ASD (see [4]). The team developing the tool was working with a large group of ASD users across three countries within the whole project duration in order to ensure that the tool will meet their diverse requirements and to evaluate the tool's effectiveness in improving reading comprehension.

The ASD users' engagement encompassed the whole project development and aimed to organize the desired assistive elements in a clear structure for the work packages involved in the development of the tool based on user requirements and to define in more precisely some important aspects for the development of the tool.

Clinicians from three different countries also took part in designing of the tool in order to create the most suitable user interface for people with ASD. The engagement of accessibility specialists played an important role in designing the UI. The result is intuitive, accessible, well-structured and responsive interface, suitable for both desktop and mobile devices.

The UI is created according to the findings and recommendations in the paper User Interface for People with Autism Spectrum Disorders (see [3]). The research extracted guidelines for the look and feel of the interface desired by the ASD users. The first prototype of the software, presented in this article, is created on the basis of this information.

\section{Interface Features}

The main features of the user interface are:

- Accessibility this design approach provides optimal view user experience easy reading and navigation with minimal efforts for resizing and scrolling. 


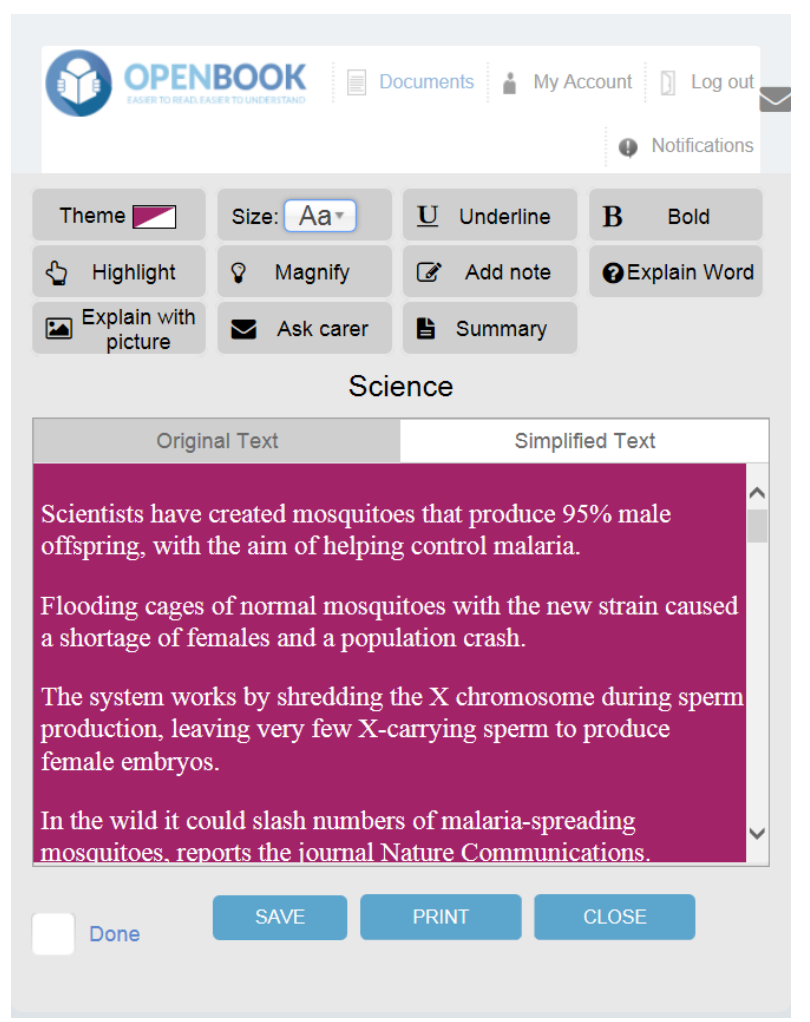

Figure 1: Demonstration of Open Book's responsive design

The buttons are kept as large as possible which enables easy navigation and work with the system (see [5]).

- Responsive user interface to support for devices with various form-factors - having in mind the increasing demands for tablets and mobile phones, UI is supported for a wide range of devices from desktop computer monitors to mobile devices. The web site supports a minimum of 800x600 desktop resolution, although we recommend using at least $1024 \times 768$. The support for mobile devices is maintained at an optimal quality at 480px and above screen width.

- Personalization - font size, style and color, background theme and menu tools can be adjusted according to user's personal needs and preferences. 


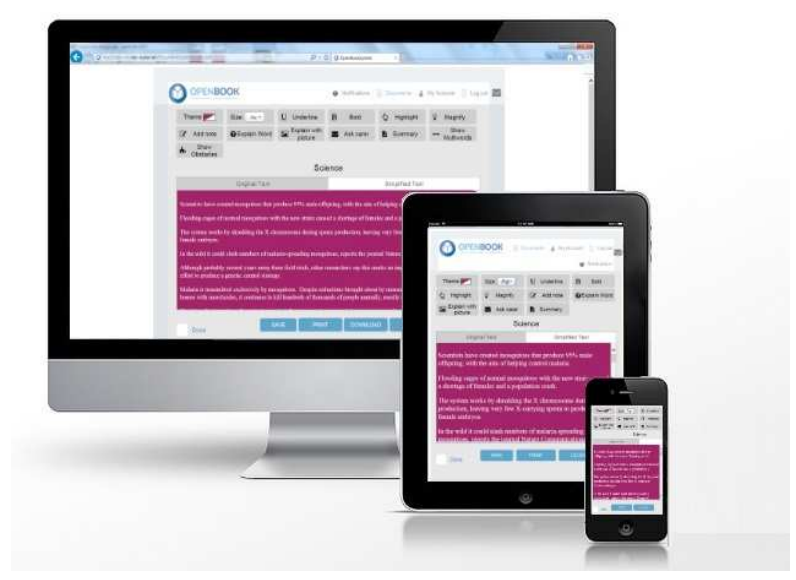

Figure 2: Support for desktop and mobile devices

\section{Design Implementation}

Based on the findings and conclusions made in the previous article, the first prototype of the Open Book software system implemented all components described. Its main goals are simple and clear design with minimum visual distractors, intuitive navigation, combination of pictures and words, simple graphics, clear fonts, large buttons, mild colors etc. which can be seen in the following examples.

\section{Sign Up}

Long and large fields where user can enter his personal information are amongst the main requirements for a registration form for users with disabilities. Only most basic information (name, e-mail and password) is required here to complete the registration successfully. Big spaces are provided between the text fields, so each field is clearly separated from the rest. Linear layout is used for the registration form in order to keep user's attention focused on a single text column. Only the picture, which is not a requirement for a successful registration, can be found on the right side of the page. 


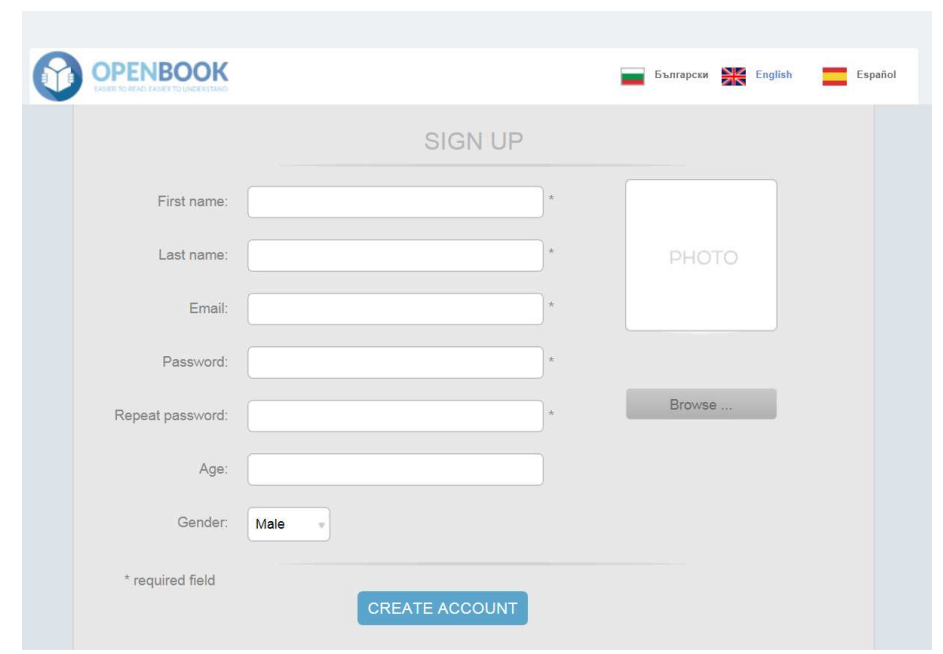

Figure 3: Sign Up Screenshot

\section{Simplified Document}

The simplification page has simple and clear design which contains minimum visual distractors. Soft, mild colors and simple graphics are used for the presentation of the main options. The reading area occupies significant space in this page presenting the simplified text to the user in in a large text box clearly separated from the rest. Researches show that ASD users get distracted by more than one column of text, so original and simplified text are disposed in different tabs avoiding the option to stay next to each other in a single page and the need of sideways scrolling.

This type of interface allows users to quickly focus their attention to the text area and adapt the presentation of document to their personal preferences.

A single toolbar is provided in the simplification page as a part of user requirements. The toolbar consists of big and clear buttons with both picture and text going next to each other - helping people with ASD understand better the meaning of the command. For the options like Bold, Underline and Picture Word-like icons are used leading the users to something common.

- Theme - color themes are created after research about the color schemes that are best perceived by people with ASD. Themes correspond to the so called "colour filters" used widely by persons with autism, Asperger syndrome, dyslexia and other reading disorders to focus their attention 


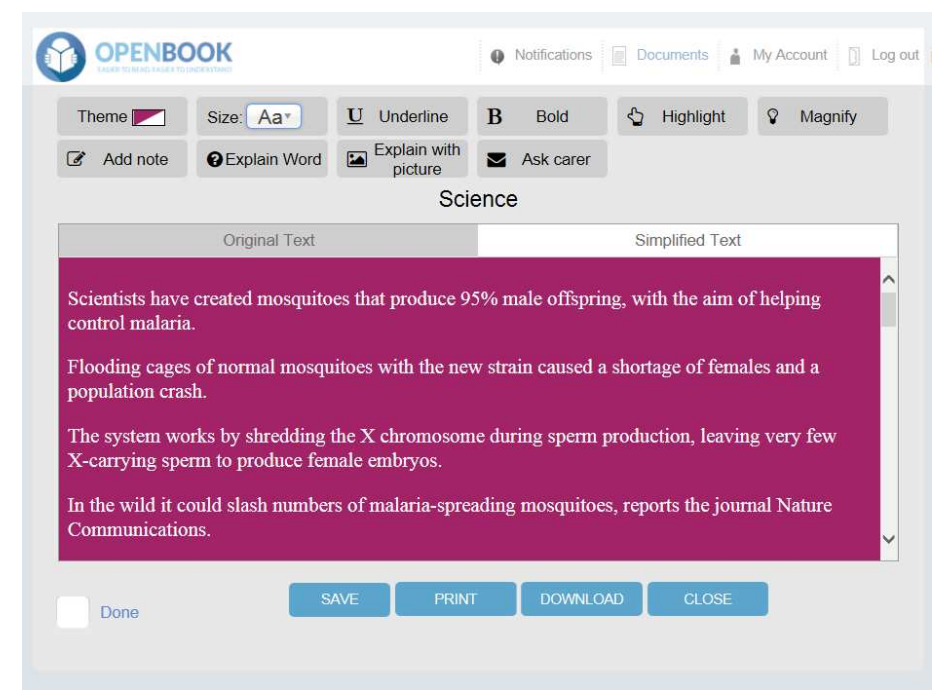

Figure 4: Simplified Document Screenshot

on the reading space. The most important feature here is the contrast between font and background. Colored filters have been found to reduce visual distortion of text in children with ASD, too (see [6]);

- Font size - the system provides 6 different sizes of font letters bigger than 12 pt due to the fact that ASD users prefer bigger letters than usual;

- Underline, Bold and Highlight are three desired options taken from Word that people with ASD often use to emphasize information that they consider important or problematic;

- Magnify option - this option helps user focus on particular sentence. People with ASD can then go through the whole text sentence by sentence taking attention on each one separately;

- Notes - users with ASD like leaving some notes whatever they do, reminding them of something important or just helping them share thoughts and feelings. Open Book provides this functionality to the users enabling them write, save, view and delete notes in the simplified document.

Trying to avoid pop-ups and other visual distractors the following UI behaviour was adopted: all messages returned by the interface and additional operations, related to the toolbar features appear in a textbox 


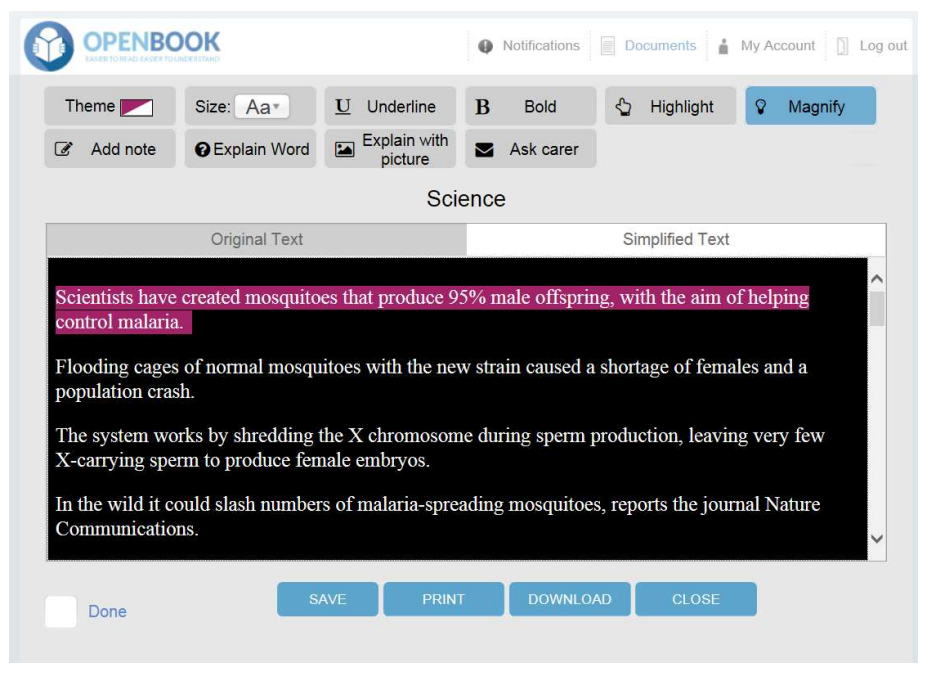

Figure 5: Demonstration of magnify option

under the main reading area. This allows user to receive all information requested meanwhile continues his work with the document.

- Explain word and explain with picture - explanation of difficult concepts with pictures and definitions is essential for improvement of reading comprehension. Researches show that visual cues stimulate better the brain activity and help users get the meaning faster and easier.

\section{Technologies Used}

\subsection{HTML5/CSS3/JS}

The application deploys various recently developed and pushed by the web community CSS3 technologies such as the new Level 3 Selectors like:

a) Selectors Level 3 - Pseudo-elements ::after, ::before; Pseudo-classes :lastchild, :not;

b) Media Queries;

c) CSS Backgrounds and Borders Module Level 3 - background-repeat, borderradius;

d) CSS Fonts Module Level 3; 


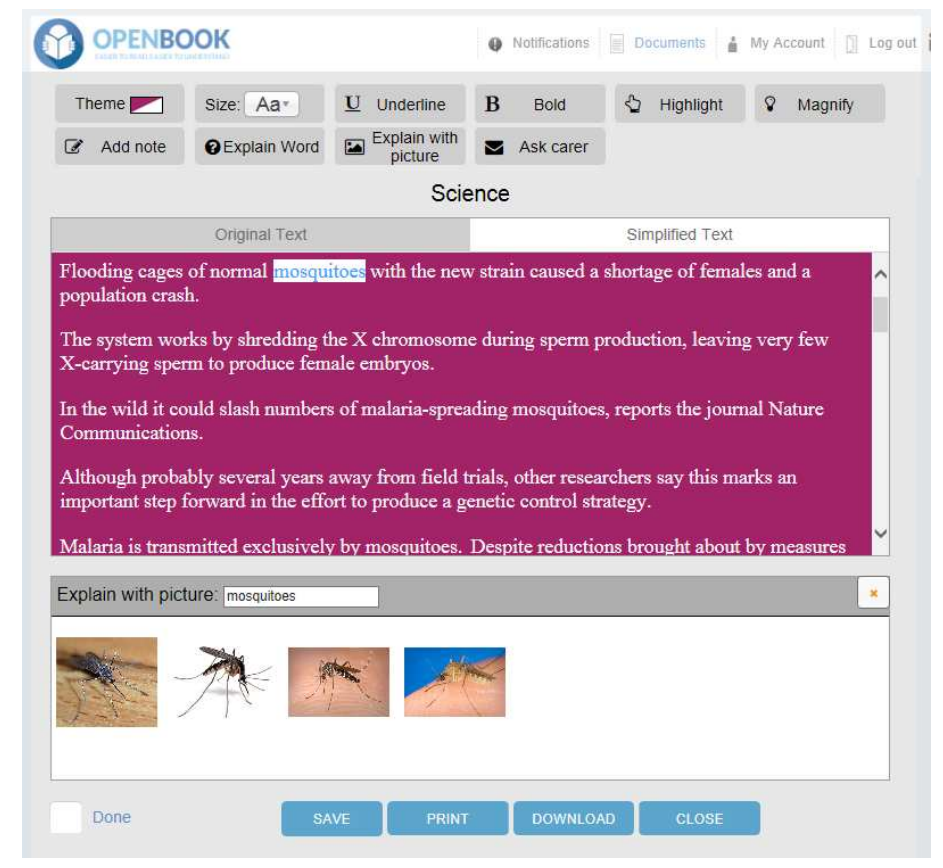

Figure 6: Demonstration of Explain with picture option

\subsection{JavaScript}

JavaScript is used extensively throughout the application mostly leveraging third-party libraries that bring to the table extremely useful tools to develop dynamic user interface, data exchange between the front-end ecosystem and the web application server as well as simply prettifying and stylizing libraries that add extra touch and feel to the whole application. The more prominent of those are:

a) jQuery - this is a very profound and powerful library which supplies a whole plethora of functionalities for traversing, selecting and modifying the DOM. It also gives the powerful AJAX wrapper for executing asynchronous JavaScript-initiated XML requests towards a remote web application or service simply by passing a JavaScript object to the jQuery.ajax function.

b) KnockoutJS - a powerful and simple to use JavaScript library for HTML binding. It uses Declarative Bindings which are mapped to a JavaScript object called "the View Model". It deploys automatic UI refresh and Dependency tracking which is extremely useful for developing business model-rich 


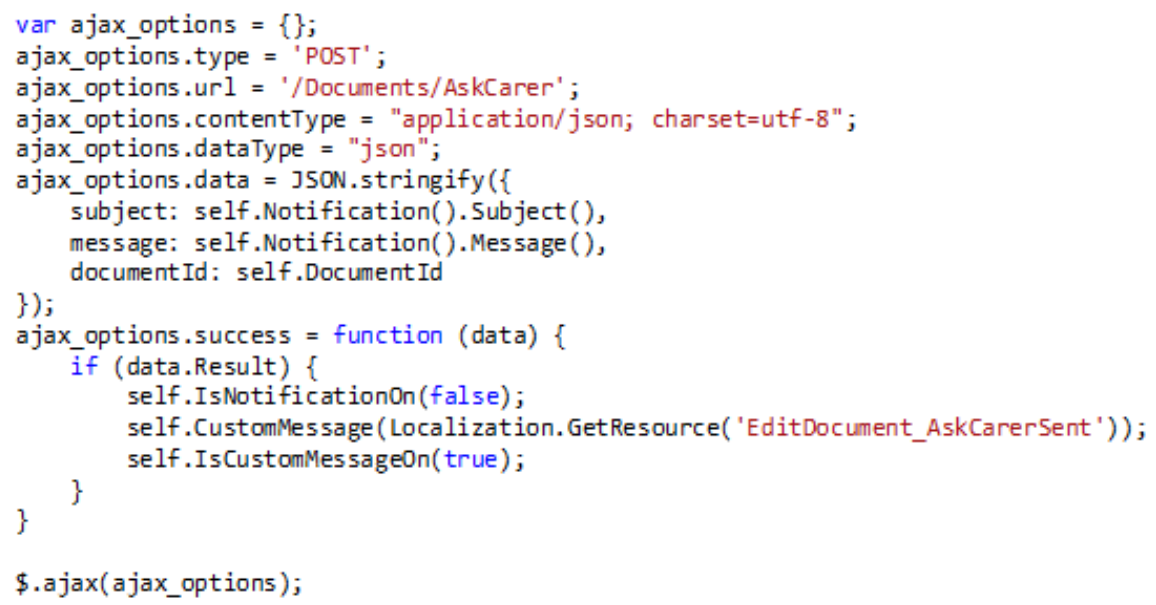

Figure 7: Initiating an ajax call

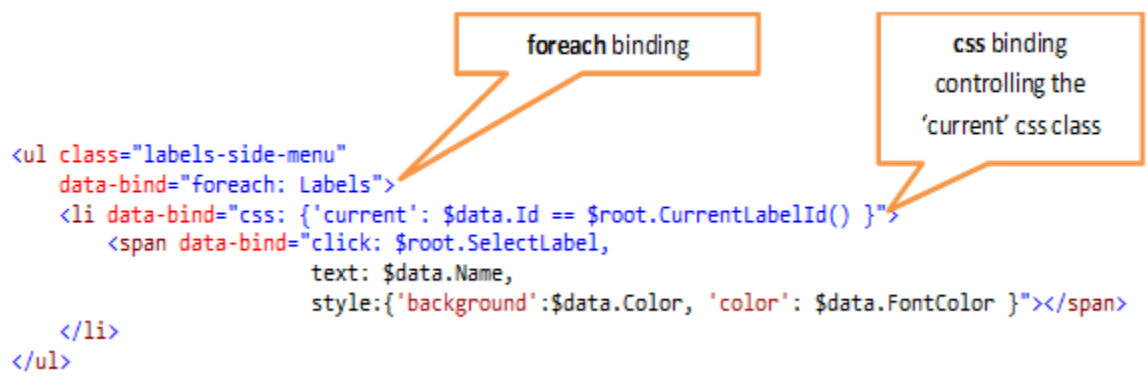

Figure 8: Declarative Knockout binding

dynamic JavaScript applications. Its declarative bindings are applied using the data-bind attribute, which maps an object or a variable of the View Model to the HTML element.

c) MomentJS - powerful library for working with Date and Time in the JavaScript environment. The native JavaScript Date() object proves insufficient and cumbersome to work with. The MomentJS library introduces a Date-wrapping object that provides a rich API for working with Date and Time

d) Chosen - simple to use library that creates and enriches the native HTML select element producing rich dropdowns and menus. 


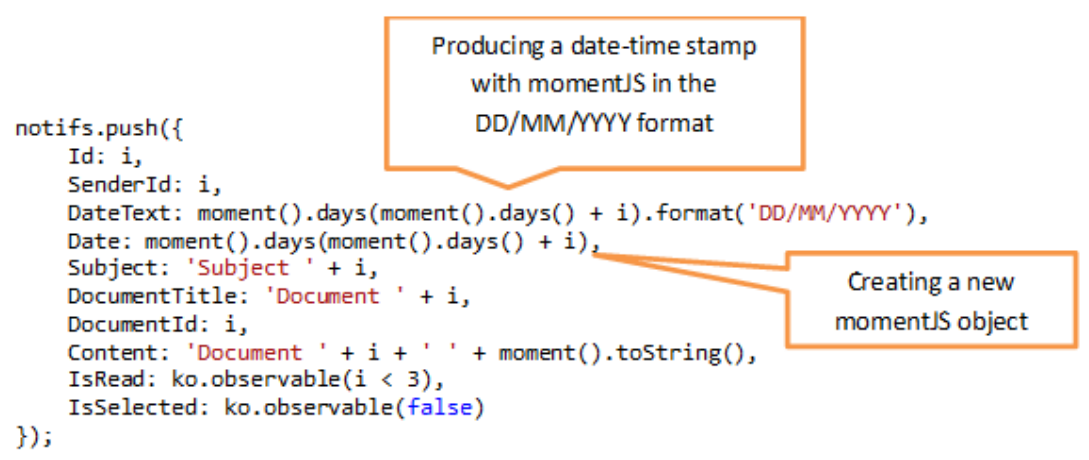

Figure 9: Using and instantiating momentJS

\subsection{Responsive User Interface}

Responsive User Interface (UI) is implemented using the@media-query css technology. This technology depends on declaring css classes and rules in different screen width ranges. Rules in those ranges overwrite rules from other classes and are considered high priority. The more commonly used width ranges are listed below:

a) @media only screen and (min-width:600px) and (max-width:960px) - rules in this section apply when the screen width is between 600 pixels and 960 pixels;

b) @media only screen and (max-width:600px) - rules in this section apply when the screen width is under 600 pixels;

\subsection{NET Framework}

NET Framework is used extensively both for back-end web request handling, serving HTML, files and data and for the Data Access layer. The OBS Web Application uses MVC 4 framework for handling web requests and the WCF framework for establishing connection with services.

a) $M V C 4^{-}$- the Web Application uses the Routing and Request handling of the MVC 4 framework which is very convenient and powerful for web applications of all types. The views used for the different pages are written using 


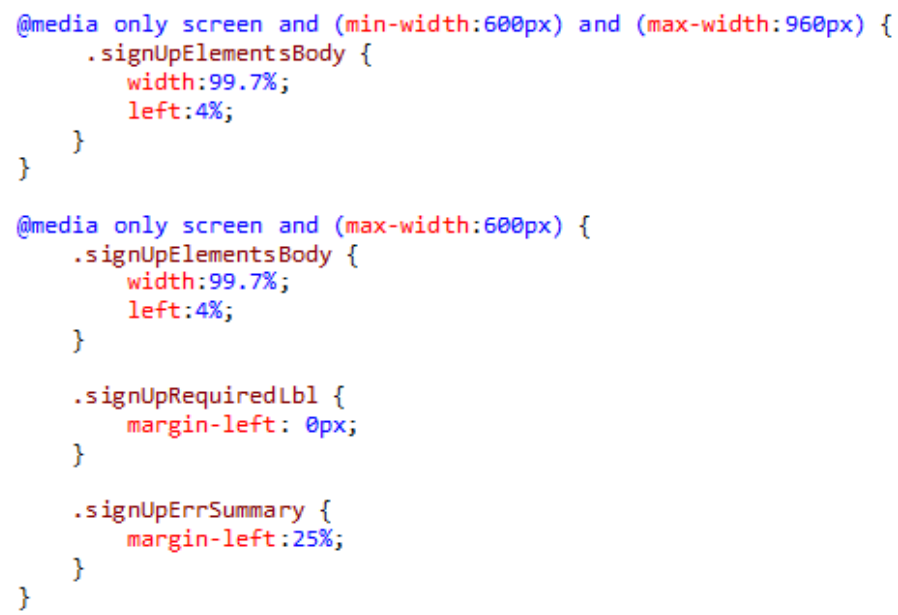

Figure 10: Sample media queries

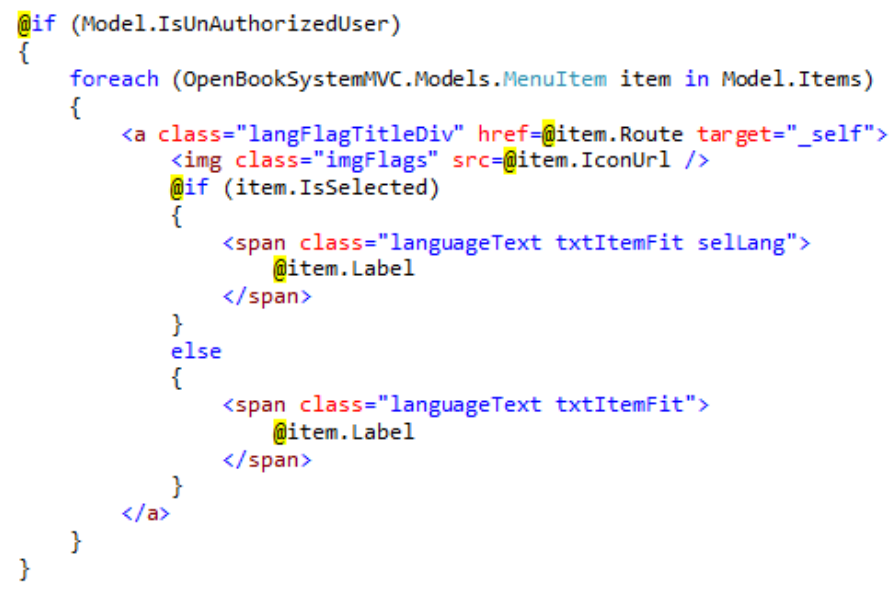

Figure 11: MVC 4 Razor View Engine

the Razor View Engine which is very powerful for constructing sophisticated HTML constructions using a C\# Model object.

b) WCF - the OBS Web Application uses the WCF (Windows Communication Foundation) for consumption of the web services. The WCF client exposes a set of methods that are consumed by the web application with the purpose of receiving and sending document or user related data for storage and processing. The calls are made using a wrapping method call: 


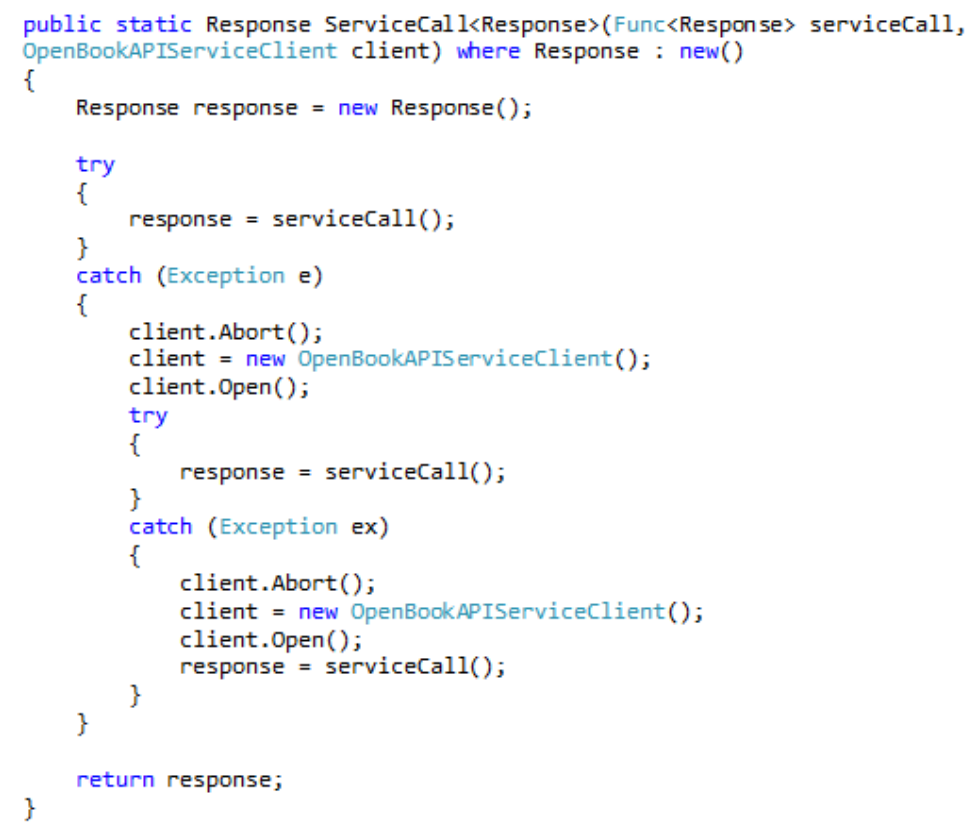

Figure 12: WCF Service call wrapper

\subsection{Browser support}

Open Book's user interface is supported on the browsers, listed below:

a) Internet Explorer $9+$

b) Google Chrome 20+

c) Mozilla Firefox $10+$

d) Safari $5+$

\section{Testing and Evaluation Results}

Detailed clinical evaluation was conducted to assess effectiveness of the Open Book software in reading comprehension of adults and children with high functioning autism. 294 people with ASD took part in the evaluation. The results clearly show that the majority of ASD users prefer simplified by Open Book texts than the original ones. When all participants (aside from controls) 
were combined together, the simplified scores were 1.2 units higher than the original scores which definitely proves that the Open Book software improves reading comprehension (see [7]).

Furthermore, feedback from caregivers of ASD people clearly proves that users find documents converted with Open Book much more interesting and engaging than before. Children spend time reading texts on topics that they will normally refuse to read. That is because they consider the Open Book documents like entertainment and playing games. Their attention is taken by the interface features, the pictures presented for complicated words and personalization settings like change of fonts, themes, etc. for much longer period than the child's normal attention span.

\section{Conclusion}

Relying on guidelines described in the article "User Interface for People with Autism Spectrum Disorders" (see [3]) the user interface of the Open Book Tool was designed and implemented. Up to now, the software have been tested and evaluated by more than 300 people. It has been used in practice for text simplification and improvement of reading comprehension by people with ASD and clinicians from three different countries.

The beta version of the system can be found on the address http:// openbooktool.net/. The official release of Open Book is planned for the end of 2014.

\section{Future Development}

Results of the FIRST project can be used to benefit other user groups except the people with ASD. Minor modifications of the system would be appropriate to adjust its settings to the preferences of other user groups like:

- People with reading impairments like dyslexia;

- Students in early grades;

- Foreign language learners; 


\section{Acknowledgements}

The project leading to this research product is partially funded by the European Commission under the Seventh Framework Programme (FP7-2007-2013) for Research and Technological Development under grant agreement \# 287607 and by the IT15-FMIIT-004 project of the Scientific Fund of the University of Plovdiv "Paisii Hilendarski", Bulgaria. This paper reflects the views only of the authors, and the Commission cannot be held responsible for any use which may be made of the information contained therein.

We would like thank all the partners from the consortium of the FIRST project, and especially the clinical partners from Deletrea, Spain for their extensive research, and Parallel World, Bulgaria for their invaluable contributions during the implementation of the user interface.

\section{References}

[1] Robert Sears, The Autism Book: What Every Parent Needs to Know About Early Detection, Treatment, Recovery and Prevention, 2009.

[2] Autism Speaks, http://www.autismspeaks.org

[3] N. Pavlov, User interface for people with autism spectrum disorders, Journal of Software Engineering and Applications, 7, No. 2 (2014), Article ID: 43152.

[4] Newsletter, Second Edition, http://www.first-asd.eu.

[5] UI Design Guidelines for Responsive Design, http://tympanus.net/codrops/2013/01/21/uidesign-guidelines-for-responsive-design/

[6] A.K. Ludlow, E. Taylor-Whiffen, A.J. Wilkins, Coloured filters enhance the visual perception of social cues in children with autism spectrum disorders, ISRN Neurol., 2012:298098 (2012), doi: 10.5402/2012/298098.

[7] A. Cerga-Pashoja, V. Jordanova, LNFT, D\%.6: Clinical Evaluation, http://firstasd.eu/?q=FIRST_documents. 
